\title{
THE USE OF LIGHT CURE IN BLEACHING TREATMENT
}

\author{
Sari DEWIYANI and Justin Gregorio PUGLISI \\ Universitas Prof. Dr. Moestopo (Beragama), Indonesia \\ *sari.drg@gmail.com
}

\begin{abstract}
Introduction: Dental aesthetic is an important need for many people. Tooth color is an important aspect to assess people's perception of beauty. Teeth whitening or teeth bleaching is an aesthetic treatment to alter the color of the teeth, which is in great demand by many patients. The use of light cure is thought to be able to improve the results of bleaching treatments. Objective: the aim of this study was to conduct an integrative review with scientific evidences relating to the use of light cure to improve bleaching treatment results. Methods: analyzing journals from the Google Scholar, National Library of Indonesia, EBSCO, PubMed, Cochrane database. The results analyzed were the value of color change in tooth after the use of light cure in bleaching treatments. Conclusion: this review reveals that the use of light cure can improve the results of bleaching treatments.
\end{abstract}

Keywords: Bleaching, Light Cure, Dental Treatment

\section{BACKGROUND}

In many metropolitan cities across the globe, dental aesthetic is an important need for many people. Tooth color is an important aspect to assess people's perception of beauty and dental aesthetic treatments are highly demanded by patients nowadays. For example, in the US it has been reported by a survey in 2018 that $41 \%$ people are not confidence with their own smile, $55 \%$ people want a smile like fashion models, and $60 \%$ people want whiter and cleaner teeth. This is because patients consider that to have an attractive smile is synonymous with health, good appearance, professional and social benefits. Dental bleaching is highly demanded treatment by patients nowadays. Compared to other restorative treatments, dental bleaching is the most conservative dental aesthetic treatment. A survey in UK, Spain, and Saudi Arabia reported that 28-34\% patients want whiter teeth and willing to do bleaching procedure. A study in Malaysia and UK also reported that dental bleaching is the most demanded treatment by patients in 2017. More than 1 million people across US spend up to 600 million dollars every year for dental treatment. In 2014, 98\% of Australians buy whitening toothpaste every 6 months. The market for whitening products and treatments are expected to rise until 2024. Most patients want whiter, easier, and faster bleaching treatment. In-Office bleaching technique is most suitable for this demand. In this technique an instrument known as light cure is frequently used. The use of such instruments is thought to increase the effectiveness of bleaching treatment, although still highly debated. Some researchers believe that the use of light cure does increase the effect of bleaching treatment, while others don't believe so, some even consider it as marketing strategy to sell more products.

The aim of this study was to conduct an integrative review with scientific evidences relating to the use of light cure to improve bleaching treatment results.

\section{Dental bleaching}

Dental bleaching is a treatment that involves oxidative chemicals which then alters the way light absorbed and reflected from material structure of tooth, thus increasing and changing the color of the tooth into whiter color. Other procedures to alter tooth color that offered by dentists are veneers and crowns, but often tooth discoloration can be fixed just by the bleaching procedure. Dental bleaching is more conservative, easy to do, and cheap compared to veneers and crowns.

\section{Bleaching materials}

Active material that is generally used in dental bleaching is peroxide compounds. Hydrogen peroxide, sodium perborate, and carbamide peroxide are the three most used peroxide compounds in dental bleaching. Hydrogen peroxide and carbamide peroxide usually used in external bleaching technique, whilst sodium perborate usually used in internal bleaching. Natrium perborate and carbamide peroxide will decompose into hydrogen peroxide when in-contact with water medium.

\section{Hydrogen peroxide $\left(\mathrm{H}_{2} \mathrm{O}_{2}\right)$}

Hydrogen peroxide generally used in in-office and at-home bleaching. In-office bleaching technique use higher concentrated hydrogen peroxide (25\% to $38 \%$ ) than in at-home bleaching ( $3 \%$ to $7,5 \%)$. Hydrogen peroxide is a strong oxidative material available in many concentration, but stable concentration of $30 \%$ to $35 \%$ are frequently used. This material must be handled carefully because of the unstable nature of peroxide, losing oxygen molecules in fast rate, and able to explode if not stored in chilled or dark container. Hydrogen peroxide is able to burn organic tissue when contact, so must be used carefully not to contact with any oral soft tissue.

\section{Carbamide peroxide $\left(\mathrm{CH}_{6} \mathrm{~N}_{2} \mathrm{O}_{3}\right)$}

Carbamide peroxide or often called urea hydrogen peroxide available in white crystal powder form which contains $35 \%$ hydrogen peroxide. Carbamide peroxide will form hydrogen peroxide and urea on liquid solution. 
This material often used in at-home bleaching technique with concentration of $10 \%$ to $20 \%$ (equivalent to $3,5 \%$ to $6,5 \%$ hydrogen peroxide). $10 \%$ carbamide peroxide will split into urea, ammonia, carbon dioxide, and 3,5\% hydrogen peroxide. This material is only used in external bleaching and able to damage surrounding hard and soft tissue. Carbamide peroxide can also affect composite resin bonding strength, thus the use of carbamide peroxide must be closely watched by dentist.

\section{Sodium perborate $\left(\mathrm{NaBO}_{3}\right)$}

Sodium perborate is available in many forms. Fresh sodium perborate contains around $95 \%$ perborate which equivalent to $9,9 \%$ oxygen. Sodium perborate has stable nature while dry, but in acidic state, warm air, or water, sodium perborate will split and create sodium metaborate, hydrogen peroxide, and new oxygen molecules. It has many forms, which are monohydrate, trihydrate, and tetrahydrate. The three forms are differentiated by their oxygen content, and thus their ability of whitening is also different. Sodium perborate is easier to handle and safer than hydrogen peroxide, and so this material is more often used in intracoronal bleaching.

\section{Bleaching methods}

There are 2 known bleaching methods, intracoronal or internal bleaching, and extracoronal or external bleaching:

\section{Intracoronal or internal bleaching}

Root canal treatment may cause tooth discoloration from within the tooth. Internal bleaching technique is chosen if the dentist wants to maintain the tooth structure. This bleaching method is more conservative compared to invasive treatments such as veneer, full crown, but the long term results are reported to be low. The indications for internal bleaching are: (1) discolorations that resulted from within pulpal space; (2) dentin discoloration; (3) discolorations that cannot be corrected by external technique. The contraindications are: (1) superficial enamel discoloration; (2) enamel defect; (3) severe dentin loss; (4) presence of caries; (5) discolored proximal composites (unless to be replaced after bleaching). There are 4 known techniques within intracoronal bleaching:

(1) Thermocatalytic technique which uses $30 \%$ to $35 \%$ natrium perborate into the pulpal space and then activated by heat. This technique is no longer used nowadays and has potential to cause external cervical root resorption from the result of irritation on cementum and periodontal ligament because of the combination between chemicals and heat (Madison and Walton 1990).

(2) Walking bleach technique which introduced by Nutting and Poe in 1961. This internal bleaching technique is the most used until today because of its safer and faster procedure. This technique requires hydrogen peroxide or natrium perborate mixed with water, heat is not used in this technique. The procedure of walking bleach is shown in picture 1 .

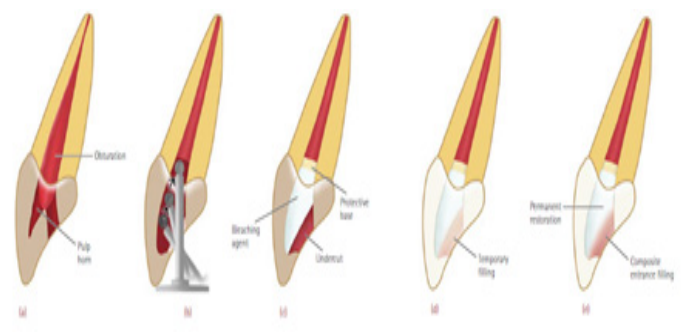

Picture 1. Complete procedure of walking bleach

(3) Combination technique where walking bleach technique is first used and then finished with external bleaching technique. This technique uses sodium perborate for the internal bleaching and $35 \%$ hydrogen peroxide as external bleaching.

(4) Ultraviolet photooxidation technique which uses ultraviolet light. $30 \%$ to $35 \%$ hydogen peroxide is placed inside pulpal space and then activated by ultraviolet light for 2 minutes, thus oxygen will be released as in thermocatalytic technique. This technique is rarely used and not recommended because it takes longer, uses higher concentration of hydrogen peroxide, and has not been proved to be more effective than walking bleach technique.

\section{Extracoronal or external bleaching}

External bleaching can be used to whiten vital or nonvital tooth. Generally, in external bleaching, hydrogen peroxide with the concentration of $30 \%$ to $35 \%$ is used and then activated by heat from a light source, heat conductor device, or laser to accelerate and to improve the result of bleaching process. There are 3 known techniques in external bleaching:

(1) In-office bleaching is best used to correct severe discoloration and uncooperative patients. Compared to at-home bleaching, in-office bleaching has several advantages, which can be directly supervised by dentists, low chance of accidental swallowed peroxide, and fewer treatment duration.

(2) At-home bleaching uses lower concentration of peroxide. $10 \%$ to $20 \%$ carbamid peroxide is often used in this technique (equivalent to $3,5 \%$ to $6,5 \%$ hydrogen peroxide). This technique can be done at home by patient themselves, but must still be supervised by dentist on regular visits. The bleaching material is placed onto special appliance that can be inserted into patient's mouth every night for 2 weeks. Compared to in-office bleaching, it can be done by patient themselves at home, safer because it uses lower concentration of peroxide, minor side effects, and cheaper, but the success of this technique is heavily depends on the patient's cooperativeness.

(3) Over-the-counter bleaching products for the past few years have increased in popularity. These products contain $3 \%$ to $6 \%$ hydrogen peroxide and can be 
used as brushes, strips, and mouth trays. Bleaching materials are available in gels, powders, and whitening tooth pastes. These products must be used in caution because some of them are not listed in food and drug administration.

\section{Bleaching mechanism}

The mechanism of bleaching remains unclear at present. However, it is generally believed that free radicals produced by hydrogen peroxide maybe responsible for bleaching effects and they are similar to that in textile and paper bleaching. Chromophore theory first introduced by Albers in 1991 is commonly used to explain bleaching mechanism. Hydrogen peroxide diffuses into email and dentin, and because its nature to be unstable, splits into hydroxils $(\mathrm{HO} \bullet)$, peridroxil radicals $\left(\mathrm{HO}_{2}^{\bullet}\right)$, superoxide anions $\left(\mathrm{O}_{2}^{\bullet-}\right)$, and reactive oxygen molecules $(\mathrm{O})$ which will then break long chained molecules into shorter chains. These shorter molecule chains will reflect light differently compared to longer molecule chains, and thus the tooth looks brighter.

\section{Light cure}

Light cure in dentistry is a device that is used to polymerized composite resin. These devices are also used on dental materials that need to be activated by hear or light. Common light cure devices that is used in dental clinics are:

\section{Ultraviolet}

Ultraviolet curing light was the first to be used in composite resin polymerization. The wavelength of this device is in the range of $364-367 \mathrm{~nm}$. It was later found that this light could cause damage to the eyes, causing cataract, and harm normal microflora inside the oral cavity. Since then this unit is no longer used in clinical practice and are no more available in the market.

\section{Tungsten halogen}

This light cure unit has been innovated to replace the ultraviolet light curing unit. Most of the units use tungsten filament halogen lamps that incorporate blue filter. This unit is able to produce light rays with the wavelength of $400-500 \mathrm{~nm}$ and is able to produce energy level up to $300 \mathrm{mV}$. The amount of time required to cure the composite is 40 seconds. High performance halogen curing light has been developed to overcome the problem of conventional halogen light that requires longer time to cure orthodontic composites. It has an $8 \mathrm{~mm}$ light guide, which generates full spectrum light filtered as blue with a range of 400 to $505 \mathrm{~nm}$. It cures orthodontic composites in eight seconds. This unit also has boost mode, which increases the light output to $1000 \mathrm{~mW} / \mathrm{cm}^{2}$. This will allow the composite under metal bracket to be cured in five seconds.

\section{Plasma arc}

This lightcure unithas been developed after the technology used by The United States Nasional Aeronautics and
Space Association in aeronautical engineering. Plasma arc light cure unit has filters that are able to narrow the spectrum of visible light to a band centered at $470 \mathrm{~nm}$. It has two electrodes with a large voltage potential that are able to ionize xenon plasma gas to emit the light, and thus the lights have an energy level of $900 \mathrm{mV}$, which is much higher than halogen lights. This unit will take only two seconds to cure orthodontic composite.

\section{Blue Light Emitting Diode (LED)}

The breakthrough in semiconductor technology has led to the use of LED in curing light cured composite resins. This unit uses indium gallium nitrate technology. As current flows through the semiconductor chips, electrical energy is converted directly into light, resulting into stable, efficient, and long lasting output of blue light with little energy emitted as heat. The spectrum of light produced is in the range of 430 to $490 \mathrm{~nm}$. It is able to cure orthodontic composite in between 10 to 40 seconds.

\section{Argon laser}

Argon laser curing unit has utilized the laser technology which provides sources that emit high intensity light within the energy band required by the initiator in light cured composite resins. Argon laser emits bluegreen light spectrum with the wavelength range of 454-496nm. Argon laser's waves are coherent, thus the photons are in phase with one another and do not collide with each other as in other light cure units. The time required to cure orthodontic composites is five seconds.

\section{Light cure mechanism}

Lights from light cure units give energy in specific spectrum. Photoinitiator molecules inside composite resin or other materials will absorb the energy and initiate chemical reaction releasing free radicals, thus the polymerization process can happen. This process is known as light polymerization.

\section{METHODS}

This literature review was made by analyzing reference sources with keywords of bleaching, light cure, and dental treatment from journals and textbooks which can be accessed from Google Scholar, PERPUSNAS RI, EBSCO, PubMed, and Cochrane database. The referenced journals are accredited and unaccredited case controls, case reports, experimental and descriptive from the year 2010 to 2020.

\section{DISCUSSION}

There has been a lot of research on the use of light cure in bleaching treatment, and also a lot of debate along with it. Bhutani N, et al (2016) in their study about evaluation of bleaching efficacy of $37,5 \%$ hydrogen peroxide on 30 human teeth using halogen, laser, and no light activation revealed that the group of teeth receiving bleaching procedure with halogen light activation 
produced the whitest teeth, followed by laser activated group, and lastly the no light activation group right after the bleaching procedure. There was significant change of color difference right after, 1, 2, and 3 weeks postbleaching procedure between halogen activated group and control group, and between laser activated group and control group. However, there was no significant difference between halogen activated group and laser activated group as shown in table 1 .

Table 1. Intra and inter group mean shade values comparison

\begin{tabular}{lccc}
\hline \multicolumn{1}{c}{ Period } & No Light & $\begin{array}{c}\text { Halogen } \\
\text { activated }\end{array}$ & $\begin{array}{c}\text { Laser } \\
\text { activated }\end{array}$ \\
\hline Before Bleaching & $13,70 \pm 3,09$ & $13,30 \pm 2,45$ & $13,20 \pm 2,36$ \\
Post Bleaching & $9,40 \pm 2,59$ & $4,90 \pm 3,38$ & $5,60 \pm 3,41$ \\
1 week & $10,30 \pm 2,79$ & $4,80 \pm 2,53$ & $6,30 \pm 3,30$ \\
2 weeks & $12,20 \pm 2,66$ & $5,80 \pm 2,35$ & $7,30 \pm 2,58$ \\
3 weeks & $12,20 \pm 2,66$ & $7,30 \pm 3,06$ & $8,30 \pm 3,40$ \\
\hline
\end{tabular}

Halogen activation was able to produce the brightest bleaching result. This might be because of the difference in the power density of both activation sources, leading to variation in the degree of heat produced in the bleaching gel which in turn enhances the decomposition of hydrogen peroxide. Durability of bleaching results was found to be maintained throughout. Durability of bleaching results was found to be maintained throughout the trial period of 3 weeks for halogen activated group. However, for laser activated group, the effect of bleaching therapy diminished over a period of 2 weeks and for no light activation group, the effect lasted no longer than a week after bleaching.

This result is similar with Alomari Q and Daraa E (2010), which they reported in their study comparing efficacy between four different methods of bleaching activation (no light activation, halogen, LED, and metal halide). They reported that halogen activated bleaching procedure was able to produce the brightest teeth. However, LED and metal halide activated bleaching procedure did not show a significant change compared to no light activation group. This might be because LED and metal halide light don't have enough energy, and so not much heat is transferred into the bleaching materials to accelerate the decomposition of hydrogen peroxide. All groups relapsed after 1 month of trial period and did not show a significant color difference between each groups. The writer concluded that the use of halogen blue light in bleaching procedure was only effective for short term result and did not affect long term result.

Kwon SR, et al (2015) did a study about the effect of light activation in bleaching procedure on different artificially stained teeth. This study concluded that the use of LED was able to produce brighter teeth than control group, especially on yellow-stained samples. This might be because the LED that was used in the study was set to high intensity $\left(466 \mathrm{~nm}, 190 \mathrm{~mW} / \mathrm{cm}^{2}\right)$, and so was able to produce enough energy to accelerate the decomposition of hydrogen peroxide. The yellow-stained samples showed a better effect in the context of light activation than the nonstained and blue-stained samples, this might be because the yellow stain might be better at absorbing energy transferred from the blue light source and thus may be susceptible to tooth whitening by hydrogen peroxide. Yellow and orange stains have been reported to respond well to tooth whitening, whereas greyish-blue stains have not.

However, some studies did not find that the use of light cure improves dental bleaching result. Nutter BJ, et all (2013) in their study about comparison between bleaching treatment with light activated and no light activated showed that there was no significant color change difference between the two groups. This study used 22 patients that was divided into two groups. First group with the total of 11 patients was treated with light activated bleaching with $25 \%$ hydrogen peroxide. Second group with the total of 11 patients was treated with no-light activated bleaching with $25 \%$ hydrogen peroxide. The color changes were measured before, right after, 1, and 2 weeks post bleaching treatment. The results of this study is shown in table 2 and picture 2 .

Table 2. Intra and inter group mean shade values comparison

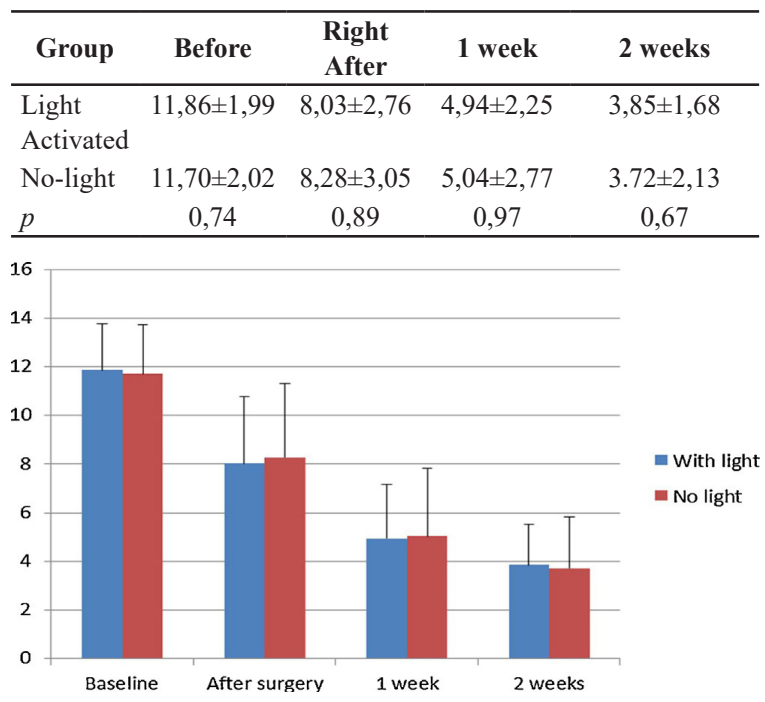

Picture 2. Change in shade for both groups at each time point

According to the result of the study, there was no significant color difference between both groups right after, 1, and 2 weeks post bleaching treatment. Both groups showed significant color change from the initial color. The light activated group showed lighter color change right after the procedure in comparison with the no-light group but nothing significant. This study did not find that the use of light cure affect bleaching treatment result. This might be because the color measurements were done manually by operator, so the assessments are heavily depending on the experience of the operator. Secondly, the color measurements were not done in the same lighting environment, and thus it may affect the tooth color perceptions from the eyes.

A study done by Almeida LC, et al (2010) showed that the use of halogen and combination of LED with 
laser did not improve in-office bleaching result. The color measurements were done before, 1 week, 3 weeks, 1 month, and 6 months after bleaching procedure with manual shade guide, during the same lighting environment, and the operators were blinded to the intervention. The halogen light that was used had the intensity of $400 \mathrm{~mW} /$ $\mathrm{cm}^{2}$, wave length of 450-500 $\mathrm{nm}$. The LED had the intensity of $120 \mathrm{~mW} / \mathrm{cm}^{2}$ and wave length of $470 \mathrm{~nm}$. The laser had the wave length of $808 \mathrm{~nm}$ and $0,2 \mathrm{~W}$ potential. This study was very similar to Goncalves RS, et all (2010), which also showed that the use of halogen, LED, and combination of LED with laser did not improve bleaching result. The color measurements were done before, 1 , and 2 weeks after bleaching procedure. Both studies did the first color change measurement one week after the bleaching procedure, not right after the procedure. This is because both writers believe that right after bleaching with the help of light activation was done, the teeth are in dehydrated state. Thus giving the effect of lighter colored teeth. This factor might be the reason why the results were different with previous studies.

\section{CONCLUSION}

Dental bleaching is a treatment that involves oxidative chemical which then alters the way light absorbed and reflected from material structure of tooth, thus increasing and changing the color of the tooth into whiter color. According to previous studies, the use of light cure might improve the effect of dental bleaching. However, its use is only able to improve short-term results and does not affect the long-term results. The light cure that might be able to improve the effect of dental bleaching must have enough energy and wavelength to accelerate hydrogen peroxide decomposition process. Thus, the best light cure to improve dental bleaching result is blue halogen light cure, and best used on yellow stained teeth.

\section{REFERENCES}

Almeida LC, Riehl H, Santos PH, Sundfeld ML, Briso AL. (2012). "Clinical Evaluation of the Effectiveness of Different Bleaching Therapies in Vital Teeth". Int J Periodontics Restorative Dent, Vol. 32(3), 303-309.

Alomari Q, Daraa EE. (2010). “Randomized Clinical Trial of In-Office Dental Bleaching with or without Light Activation". The Journal of Contemporary Dental Practice, Vol. 11(1), 17-24.

Alqahtani, MQ. (2014). "Tooth-Bleaching Procedures and Their Controversial Effects: A Literature Review". The Saudi Dental Journal, Vol. 26(2), 33-46.

Alsheikh R, Elembaby A. (2018). "Spectrophotometric Comparison of Effectiveness of Two In-office Bleaching Agents with/without Light Activation: A Clinical Study". The Journal of Contemporary Dental Practice, Vol. 19(6), 637-641.
Bhutani N, Venigalla BS, Patil JP, Singh TV, Jain A. (2016). "Evaluation of Bleaching Efficacy of 37.5\% Hydrogen Peroxide on Human Teeth Using Different Modes of Activations: An in vitro study". J Conserv Dent, Vol. 19, 259-263.

Calderini A, Sciara S, Semeria C, Pantaleo G, Polizzi E. (2016). "Comparative Clinical and Psychosocial Benefits of Tooth Bleaching: Different Light Activation of a $38 \%$ Peroxide Gel in a Preliminary Case-Control Study". Clin Case Rep, Vol. 4(8), 728-735.

Gonçalves RS, Costa CAS, Soares DGS, dos Santos PH, Cintra LTA, Briso ALF. (2016). "Effect of Different Light Sources and Enamel Preconditioning on Color Change, H2O2 Penetration, and Cytotoxicity in Bleached Teeth". Operative Dentistry, Vol. 41(1),83-92.

Ingle Jl, Bakland LK, Baumgartner JC. (2019). Ingle's Endodontics. $7^{\text {th }}$ ed. DC Decker, Hamilton, Canada.

Izidoro ACSA, Martins GC, Higashi C, Zander-Grande C, Tay LY, Gomes JC, Campanha NH, Jorge JH. (2015). Combined Technique for Bleaching NonVital Teeth with 6-Month Clinical Follow-Up: Case Report. Int J Oral Dent Health, Vol. 1(2), 009.

Krishna VG, Chandra BS. (2010). Grossman's Endodontic Practice. $12^{\text {th }}$ ed. Wolters Kluwer, Gurgaon, India.

Kwon SR. Tooth Whitening with Peroxides. In: Perdigão J (Editor). (2016). Tooth Whitening: An EvidenceBased Perspective. Springer, Minneapolis, America.

Kwon SR, Kurti SR, Oyoto U, Li Y. (2015). "Effect of Light-Activated Tooth Whitening on Color Change Relative to Color of Artificially Stained Teeth: Effect of Light-Activated Tooth Whitening on Color Change". Journal of Esthetic and Restorative Dentistry, Vol. 27(S1), S10-S17.

Nutter BJ, Sharif MO, Smith AB, Brunton PA. (2013). "A Clinical Study Comparing the Efficacy of Light Activated in-Surgery Whitening Versus in-Surgery Whitening without Light Activation". Journal of Dentistry, Vol. 41(5), e3-e7

Rueggeberg FA, Giannini M, Arrais CAG, Price RBT. (2017). "Light Curing in Dentistry and Clinical Implications: A Literature Review". Braz Oral Res, Vol. 31, 64-91.

Sherwood A. (2010). Essentials of Operative Dentistry. Jaypee Brothers Medical, New Delhi, India.

Soesilo D. (2016). "Perawatan Internal Bleaching Untuk Estetik Gigi Pasca Perawatan Endodontik”. DENTA Jurnal Kedokteran Gigi, Vol. 10(2), 1-6.

Torabinejad M, Walton R. (2020). Endodontics: Principle and Practice. $6^{\text {th }}$ ed. Elsevier, St. Louis, America. 